\title{
Iron deficiency is common in idiopathic pulmonary arterial hypertension
}

\author{
G. Ruiter*\#, S. Lankhorst*,\# , A. Boonstra*, P.E. Postmus*, S. Zweegman ", \\ N. Westerhof ${ }^{\#}$, W.J. van der Laarse ${ }^{\#}$ and A. Vonk-Noordegraaf*
}

ABSTRACT: The aims of this study were to assess the prevalence of iron deficiency in idiopathic pulmonary arterial hypertension (IPAH) and investigate whether oral iron supplementation has effects in iron-deficient patients.

Iron parameters were measure for all IPAH patients attending our centre (VU University Medical Center, Amsterdam, the Netherlands) between May 2009 and February 2010. Iron data were related to clinical parameters, including 6-min walking distance (6MWD), and haemodynamic parameters measured during right heart catheterisation. In a subset of iron-deficient patients, the uptake of iron from the bowel was studied after administering oral iron for $\mathbf{4}$ weeks.

Iron deficiency was found in $30(43 \%)$ out of 70 patients. 6MWD was reduced in iron-deficient patients compared with iron-sufficient patients (mean \pm SD $390 \pm 138$ versus $460 \pm 143 \mathrm{~m} ; \mathrm{p}<0.05$ ) irrespective of the existence of anaemia. In a subset of 18 patients that received oral iron, ferritin levels were significantly increased, although eight patients only slightly increased their iron storage.

This study shows that iron deficiency is frequently present in IPAH and is associated with a lower exercise capacity. The small response to oral iron in $44 \%$ of the treated patients suggests impaired iron absorption in these patients.

\section{KEYWORDS: Exercise capacity, iron deficiency, pulmonary hypertension}

I ron is an important cofactor in oxygen transport; it is needed to produce haemoglobin in erythrocytes, and myoglobin in the heart and skeletal muscles, which facilitates oxygen diffusion and oxygen storage. Moreover, iron functions in several mitochondrial oxidative enzymes and the respiratory chain. Consequently, when iron deficiency occurs, oxygen supply to the heart and skeletal muscles is reduced, resulting in decreased exercise performance [1-5].

Iron deficiency is frequently encountered in chronic left heart failure [6-9]. The cause of iron depletion is thought to be inflammationmediated and/or due to renal failure. Both conditions cause an increase in hepcidin concentration (increased production in the liver or reduced breakdown by the kidney), which causes a downregulation of iron release from storage sites and decreased uptake of iron from the gut mucosa [10-13]. It has also been shown that restoring iron levels improves quality of life and New York Health Association (NYHA) functional class in iron-deficient patients with chronic left heart failure [6-9]. In contrast to iron deficiency in left ventricular failure, no clinical data exist for right ventricular failure, although KRASUSKI et al. [14] reported that anaemia in pulmonary hypertension decreased survival. Pulmonary hypertension is the most common cause of right ventricular failure. Since iron is important in increasing oxidative metabolism of the hypertrophied right ventricle to cope with the increased afterload and for skeletal muscles to perform physical exercise, iron deficiency might thus have detrimental effects in pulmonary hypertension. However, data are lacking about the prevalence and effect of iron deficiency in right heart failure.

Therefore, we performed a study that aimed to determine the prevalence of iron deficiency in idiopathic pulmonary arterial hypertension (IPAH) patients. In addition, we compared iron-deficient with iron-sufficient IPAH patients to investigate whether iron deficiency is related to disease severity and exercise capacity. Finally, we studied whether digestive uptake of oral iron is impaired in iron-deficient IPAH patients.

\section{METHODS}

In this observational descriptive study, iron status was determined for all consecutive IPAH patients attending the VU University Medical
AFFILIATIONS

Depts of *Pulmonology,

\#Physiology, and

"Haematology, VU University

Medical Center, Amsterdam,

The Netherlands.

CORRESPONDENCE

A. Vonk-Noordegraaf

Dept of Pulmonology

VU University Medical Center

de Boelelaan 1117

1081 BV Amsterdam

The Netherlands

E-mail: a.vonk@vumc.nl

Received:

June 302010

Accepted after revision:

Sept 162010

First published online:

Sept 302010 
Center outpatient clinic (Amsterdam, the Netherlands) for their annual or 4-month check-up visit, and all hospitalised IPAH patients between May 2009 and February 2010. Haemoglobin $(\mathrm{Hb})$, haematocrit $(\mathrm{Ht})$, mean corpuscular volume $(\mathrm{MCV})$, kidney function and $\mathrm{N}$-terminal pro-brain natriuretic peptide (NT-proBNP) are routinely measured during every visit in our clinic. Assessment of iron status, including serum iron, transferrin saturation, total iron binding capacity (TIBC) and serum ferritin levels, was performed from the same material of the routinely drawn blood samples. $\mathrm{Hb}$ and $\mathrm{MCV}$ were measured spectrophotometrically (Cell-Dyn Sapphire; Abbott, Hoofddorp, the Netherlands), with Ht calculated from the product of MCV and erythrocyte number. Serum ferritin and NT-proBNP were determined using sandwich immunoassays with electrochemical luminescence technology (Modular E170 system; Roche, Almere, the Netherlands). Serum creatinine, iron and TIBC were measured using photometry, and transferrin saturation was calculated from serum iron divided by TIBC (Modular P800 system; Roche).

Iron deficiency was defined as serum iron $<10 \mu \mathrm{mol} \cdot \mathrm{L}^{-1}$ and decreased transferrin saturation $(<15 \%$ in females and $<20 \%$ in males) irrespective of the coexistence of anaemia $(\mathrm{Hb}$ $<12 \mathrm{~g} \cdot \mathrm{dL}^{-1}$ in females and $<13 \mathrm{~g} \cdot \mathrm{dL}^{-1}$ in males) $[8,9,15]$. Serum ferritin was not used to define iron deficiency because of possible false-high values due to increased inflammatory markers.

In cases of iron deficiency, patients were questioned about pathological blood loss (gastrointestinal or menstrual), known haematological comorbidities and previous anaemia to obtain information about possible causes for the iron deficiency. Standard oral iron treatment was started in the newly diagnosed iron-deficient IPAH patients as part of good clinical practice. These patients $(n=21$; all females with or without anaemia) received oral iron (200 $\mathrm{mg}$ ferrous fumarate t.i.d. for 4 weeks; Teva Pharmaceuticals Industries, Utrecht, the Netherlands). Patients were encouraged to complete these 4 weeks of treatment through telephone calls from specialised pulmonary hypertension nurses. After 4 weeks of treatment, iron parameters were measured again.

Iron data were related to simultaneously measured clinical parameters (age, sex, NYHA functional class, body mass index (BMI), current treatment and NT-proBNP). Haemodynamic parameters from right heart catheterisation (mean pulmonary artery pressure, mean right atrial pressure, cardiac output, pulmonary vascular resistance, and arterial and mixed venous saturation) and 6-min walking distance (6MWD) were obtained $<1$ yr before iron measurement.

The study protocol was approved by the Institutional Review Board on Research Involving Human Subjects (Amsterdam, the Netherlands) and it was stated that this protocol did not require informed consent, as all measurements were performed within the routine measurements and oral iron was given after clinical indication.

\section{Statistical analysis}

In order to compare parameters between iron-deficient and iron-sufficient patients, normal distribution of the data was tested with D'Agostino-Pearson testing and unpaired t-tests were performed. Paired t-tests were used to determine the effects of oral iron and nonparametric Mann-Whitney U-tests were performed to compare iron-deficient anaemic with irondeficient nonanaemic patients. In order to compare categorical data, Fisher's exact tests or Chi-squared tests were performed. All data are presented as mean $\pm \mathrm{SD}$, unless otherwise stated, with a p-value $<0.05$ taken as statistically significant. Analyses were performed with SPSS 15.0 (SPSS Inc., Chicago, IL, USA).

\section{RESULTS}

Iron parameters and baseline characteristics for $70 \mathrm{IPAH}$ patients (15 males and 55 females) are shown in table 1 . The haemodynamic characteristics of the patients showed that the patients had pulmonary hypertension with a mean \pm SD pulmonary artery pressure of $50.2 \pm 14.1 \mathrm{mmHg}$ and a pulmonary vascular resistance of $786 \pm 500 \mathrm{dyn} \cdot \mathrm{s} \cdot \mathrm{cm}^{-5}$. All patients were treated with pulmonary hypertension medication and 61 patients used oral anticoagulants. Iron deficiency was found in $30(43 \%)$ patients. None of these patients reported current or past pathological gastrointestinal or abnormal gynaecological blood loss. The female patients were pre- $(n=28)$ or postmenopausal $(n=27)$ with a mean age of 39.1 and 62.9 yrs, respectively. There were no significant differences in iron deficiency incidence between these groups: of the pre-menopausal patients, $50 \%$ were iron-deficient versus $44 \%$ in the postmenopausal group $(\mathrm{p}=0.8)$.

Right heart catheterisation information and 6MWD data were available in 67 (39 iron-sufficient and 28 iron-deficient patients) and 68 patients (40 iron-sufficient and 28 iron-deficient patients), respectively. In two patients, no $6 \mathrm{MWD}$ data were available because one patient declined to perform the 6MWD in recent years and data were missing for the second patient. As presented in table $1, \mathrm{Hb}$ and $\mathrm{Ht}$ were significantly lower in the iron-deficient patients, although anaemia was only present in 12 out of 30 iron-deficient patients. There were no significant differences in haemodynamic data between the iron-deficient and the non-sufficient groups. However, 6MWD was significantly lower in iron-deficient patients compared with ironsufficient patients $(390 \pm 138$ versus $460 \pm 143 \mathrm{~m}$; $\mathrm{p}<0.05)$, but similar in iron-deficient anaemic $(n=12)$ and nonanaemic $(\mathrm{n}=18)$ patients $(397 \pm 132$ versus $385 \pm 147 \mathrm{~m}$; $\mathrm{p}=0.82)$ (fig. 1$)$. There was no difference in the clinical and haemodynamic parameters between iron-deficient anaemic and nonanaemic patients.

\section{Oral iron uptake in iron-deficient IPAH patients}

Of the 21 patients who received oral iron treatment, 18 patients completed the 4 weeks of treatment. The other three patients did not complete the course due to side-effects secondary to oral iron intake consisting of gastrointestinal constipation and nausea. Baseline characteristics of the 18 patients showed that they were representative for the iron-deficient group.

In figure 2, iron parameters are shown before and after 4 weeks of oral iron. Only two patients increased their serum iron levels significantly and 14 patients did not reach normal transferrin saturation values ( $9 \pm 3$ versus $12 \pm 4 \%$ ). Serum ferritin levels were significantly increased after 4 weeks $\left(12 \pm 7\right.$ versus $\left.32 \pm 20 \mu \mathrm{g} \cdot \mathrm{L}^{-1} ; \mathrm{p}<0.05\right)$. Despite this overall increase in serum ferritin, a subset of the patients $(n=8 ; 44 \%)$ was not able to significantly increase serum ferritin and did not reach normal serum ferritin values $\left(11 \pm 5\right.$ versus $19 \pm 14 \mu \mathrm{g} \cdot \mathrm{L}^{-1}$; 


\section{TABLE 1 Patient characteristics}

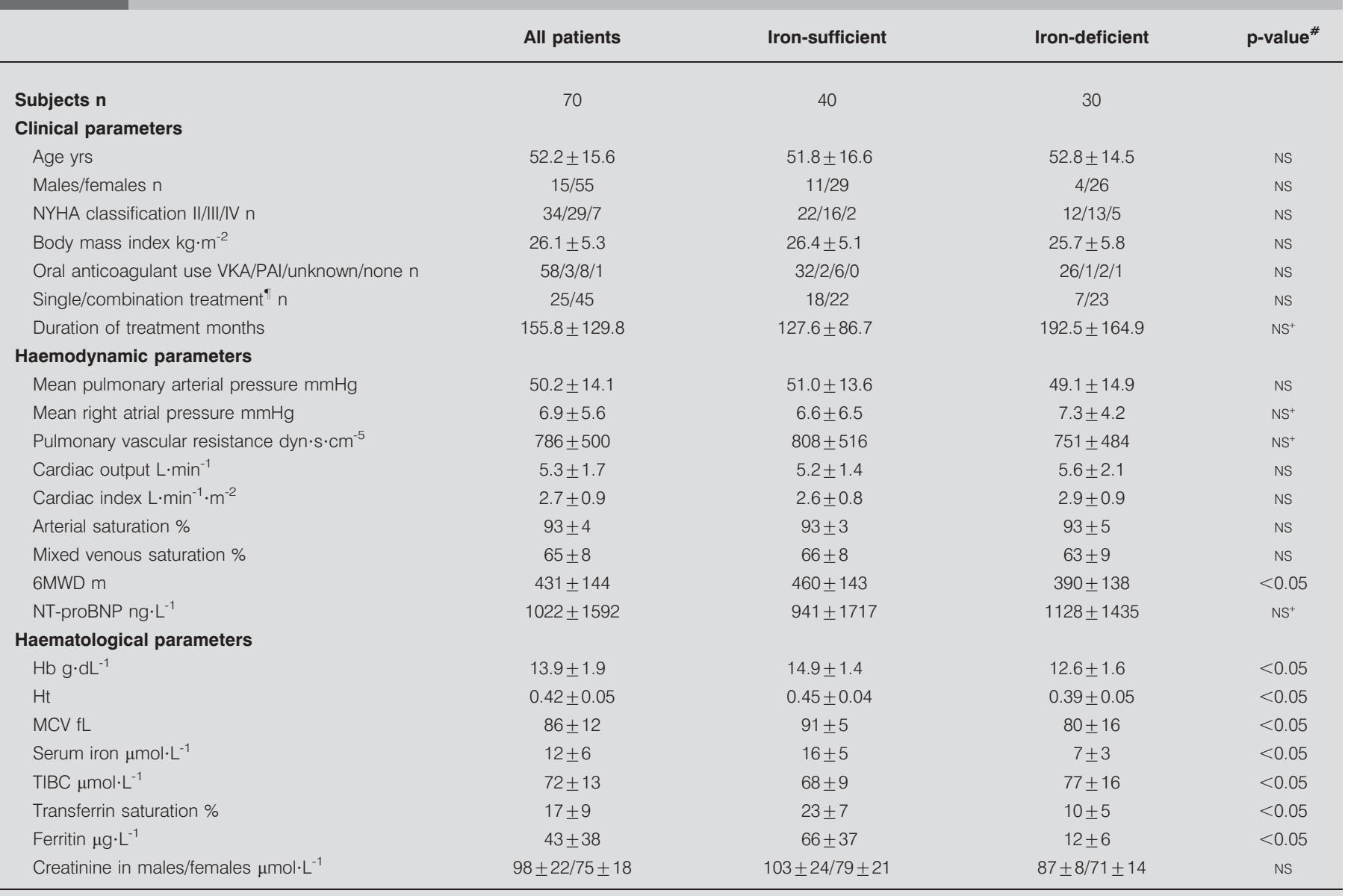

Data are presented as mean $\pm \mathrm{SD}$, unless otherwise stated. NYHA: New York Heart Association; VKA: vitamin K antagonist; PAl: platelet aggregation inhibitor; 6MWD: 6-min walking distance; NT-proBNP: N-terminal pro-brain natriuretic peptide; Hb: haemoglobin; Ht: haematocrit; MCV: mean corpuscular volume; TIBC: total iron binding capacity; NS: nonsignificant. ${ }^{*}$ : independent-sample t-testing to compare iron-sufficient with iron-deficient idiopathic pulmonary arterial hypertension patients and Fisher's exact or Chi-squared testing for categorical data; ": type of idipathic pulmonary arterial hypertension treatment (number of iron-sufficient/deficient patients) was endothelin receptor antagonists (24/19), phospodiesterase-5 inhibitor (20/26) and prostanoids (14/16); ${ }^{+}$: independent-sample t-testing after log transformation to obtain normal distribution.

a)

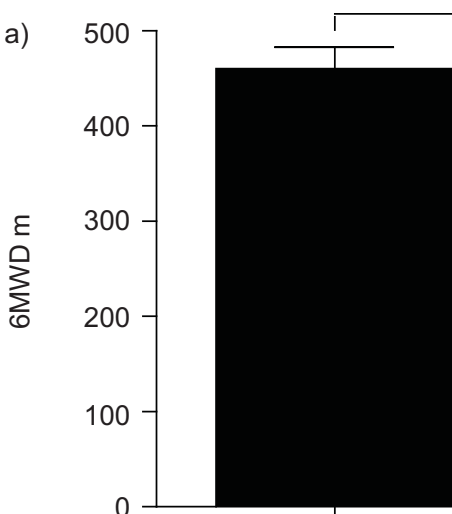

Iron sufficient
*

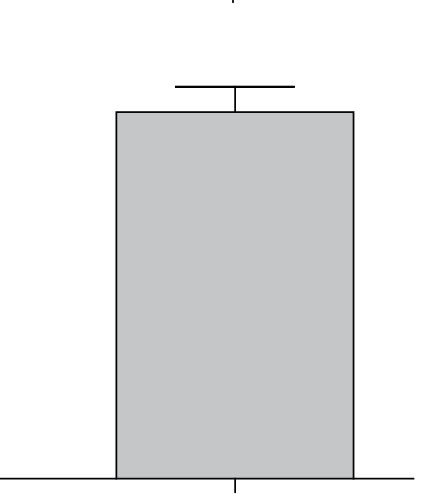

Iron deficient b)

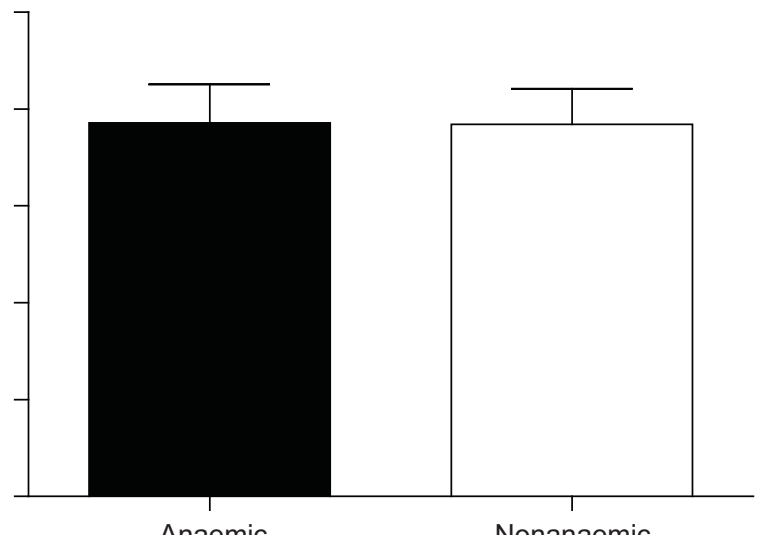

Anaemic

Nonanaemic

FIGURE 1. The 6-min walking distance (6MWD) of a) idiopathic pulmonary arterial hypertension patients with $(n=30)$ and without $(n=40)$ iron deficiency and $b)$ irondeficient patients subdivided into anaemic (haemoglobin $<12 \mathrm{~g} \cdot \mathrm{dL}^{-1}$ in females and $<13 \mathrm{~g} \cdot \mathrm{dL}^{-1}$ in males; $\mathrm{n}=12$ ) and nonanaemic $(\mathrm{n}=18)$ groups. Data presented as mean \pm SEM. *: $p<0.05$. 

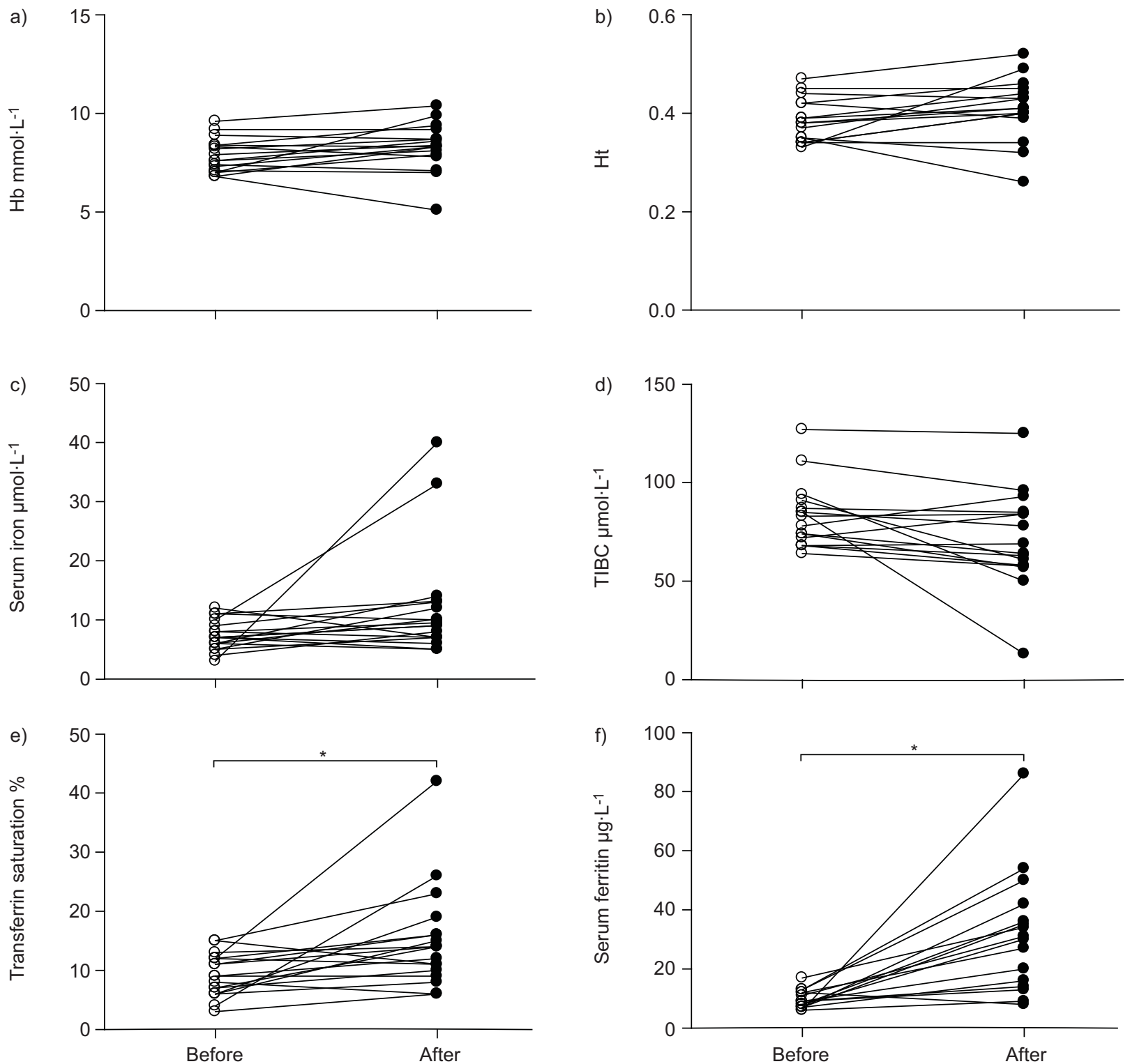

FIGURE 2. Effects of 4 weeks of oral iron on a) haemoglobin ( $\mathrm{Hb}$ ) concentration, b) haematocrit ( $\mathrm{Ht}$ ), c) serum iron concentration, d) total iron binding capacity (TIBC), e) transferrin saturation and f) serum ferritin. Each data point represents one patient with a connecting line between the values for before $(\bigcirc)$ and after $(\bullet)$ treatment $(n=18)$ $*: p<0.05$

$\mathrm{p}=0.09$ ), while the other $56 \%$ did, despite similar haemodynamic, clinical and haematological parameters.

\section{DISCUSSION}

To the best of our knowledge, this is the first study that describes iron deficiency in IPAH. We demonstrated that iron deficiency is a common feature (43\%) in IPAH patients and is associated with a decreased 6MWD regardless of the presence of anaemia. After administration of oral iron, eight out of 18 patients did not restore ferritin levels to normal values.

\section{Patients}

This study was performed in IPAH patients, as those patients have pulmonary hypertension without comorbidities that might explain iron deficiency. The population was predominantly female (15 males and 55 females); however, no significant differences were found between sexes in the irondeficient and iron-sufficient groups.

The $43 \%$ prevalence of iron deficiency in this study corresponds to data from chronic left heart failure patients, where iron deficiency prevalence varies between $15 \%$ and $61 \%$ in anaemic, and $20 \%$ and $43 \%$ in nonanaemic patients, depending on the definition of iron deficiency [16-20].

\section{Possible mechanisms of iron deficiency in IPAH}

IPAH patients can become iron deficient by reduced intake, increased loss or impaired uptake. Although BMI values were normal, we cannot exclude reduced iron intake as a cause of iron deficiency. Also, 61 patients were known to use anticoagulants, indicating that increased blood (iron) loss might be considered a possible cause, but no significant differences in 
iron deficiency incidence were found between pre- and postmenopausal females [21]. Concerning iron uptake, we found that eight out of 18 patients who received oral iron did not seem to increase serum ferritin levels as much as the other 10 patients [11]. Although doses and clinical and haemodynamic parameters were similar, iron absorption may be different between the patients, resulting in the reduced response to oral iron. This impaired iron absorption could be due to both gastrointestinal oedema, although right atrial pressures were similar, and decreased iron release from storage sites or enterocytes due to inflammation. In IPAH, interleukin (IL)-6 levels are known to be increased, and IL- 6 induces hepcidin release from the liver, which reduces iron release from iron storage cells and duodenal cells [22-26]. Whether this contributes to iron deficiency in IPAH has yet to be determined.

\section{Iron deficiency and exercise capacity}

The significantly reduced 6MWD in iron-deficient IPAH patients indicates that iron plays an important role in maintaining exercise performance. The decreased 6MWD cannot be explained by differences in disease severity, as haemodynamic characteristics of both groups were similar. However, it cannot be decided whether iron deficiency has an effect on right ventricular function, skeletal muscle function or both. Additionally, SMITH and co-workers [27, 28] showed that iron supplementation in healthy humans with iron deficiency and hypoxiainduced pulmonary hypertension at high altitude decreased mean pulmonary artery pressure. Whether iron treatment also has effects on the pulmonary vasculature in patients with IPAH is unknown. However, data from iron deficiency in left heart failure showed that administration of intravenous iron improved both quality of life and NYHA functional class [6-9]. ANKER et al. [9] also showed that 6MWD significantly increased after intravenous iron administration. The present data cannot answer the question of whether iron deficiency is a cause of more advanced symptoms or if it results from a more severe disease state. However, it appears that iron deficiency is the cause for the reduced $6 \mathrm{MWD}$, as all other parameters are similar between the iron-deficient and iron-sufficient groups. Our findings warrant further research aimed at studying the effects of restoration of iron levels, for instance by means of intravenous iron supplementation in iron deficient IPAH patients on exercise capacity.

\section{Limitations}

This study is an observational prospective study that, therefore, has several limitations. There is no information about the inflammatory status of the patients, as C-reactive protein is not routinely measured in our IPAH patients. Also, haemodynamic measurements were not measured at the same time as iron status determination. Since right heart catheterisation was not repeated, implying no change in clinical condition, we assumed that the right heart catheterisation data provided are representative of the clinical state. Additionally, NT-proBNP values obtained at the same time of iron measurement and at the time of right heart catheterisation were used as markers of disease progression. Because of the design of the study, no clinical measurements were repeated after the oral iron administration.

\section{Conclusion}

In this study, iron deficiency is described for the first time in IPAH with a prevalence of $43 \%$. The small response to oral iron in $44 \%$ of the patients suggests problems with iron absorption or release. The exact mechanisms of the iron deficiency in IPAH and whether intravenous iron administration is useful have yet to be determined.

\section{SUPPORT STATEMENT}

G. Ruiter and A. Vonk-Noordegraaf were supported by the Netherlands Organization for Scientific Research (grant number NWO-VIDI 917.96.306)

\section{STATEMENT OF INTEREST}

Statements of interest for A. Boonstra and N. Westerhof can be found at www.erj.ersjournals.com/site/misc/statements.xhtml

\section{ACKNOWLEDGEMENTS}

The authors would like to thank I. van der Mark-Gobielje, F.P.T. Oosterveer and M. Wagenaar (all VU Medical Center, Amsterdam, the Netherlands) for their help with patient inclusion and monitoring.

\section{REFERENCES}

1 Davies KJ, Maguire JJ, Brooks GA, et al. Muscle mitochondrial bioenergetics, oxygen supply, and work capacity during dietary iron deficiency and repletion. Am J Physiol 1982; 242: E418-E427.

2 Davies KJ, Donovan CM, Refino CJ, et al. Distinguishing effects of anaemia and muscle iron deficiency on exercise bioenergetics in the rat. Am J Physiol 1984; 246: E535-E543.

3 Beard JL. Iron biology in immune function, muscle metabolism and neuronal functioning. J Nutr 2001; 131: 568S-579S.

4 Haas JD, Brownlie T. Iron deficiency and reduced work capacity: a critical review of the research to determine a causal relationship. J Nutr 2001; 131: 676S-688S.

5 Brownlie T, Utermohlen V, Hinton PS, et al. Tissue iron deficiency without anaemia impairs adaptation in endurance capacity after aerobic training in previously untrained females. Am J Clin Nutr 2004; 79: 437-443.

6 Bolger AP, Bartlett FR, Penston HS, et al. Intravenous iron alone for the treatment of anaemia in patients with chronic heart failure. J Am Coll Cardiol 2006; 48: 1225-1227.

7 Toblli JE, Lombrana A, Duarte $\mathrm{P}$, et al. Intravenous iron reduces NT-pro-brain natriuretic peptide in anemic patients with chronic heart failure and renal insufficiency. J Am Coll Cardiol 2007; 50: 1657-1665.

8 Okonko DO, Grzeslo A, Witkowski T, et al. Effect of intravenous iron sucrose on exercise tolerance in anemic and nonanemic patients with symptomatic chronic heart failure and iron deficiency FERRIC-HF: a randomized, controlled, observer-blinded trial. J Am Coll Cardiol 2008; 51: 103-112.

9 Anker S, Comin Colet J, Filippatos G, et al. Ferric carboxymaltose in patients with heart failure and iron deficiency. N Engl J Med 2009; 361: 2436-2448.

10 Wrighting DM, Andrews NC. Interleukin-6 induces hepcidin expression through STAT3. Blood 2006; 108: 3204-3209.

11 Handelman GJ, Levin NW. Iron and anaemia in human biology: a review of mechanisms. Heart Fail Rev 2008; 13: 393-404.

12 van der Putten K, Braam B, Jie KE, et al. Mechanisms of disease: erythropoietin resistance in patients with both heart and kidney failure. Nat Clin Pract Nephrol 2008; 4: 47-57.

13 Babitt JL, Lin HY. Molecular mechanisms of hepcidin regulation: implications for the anaemia of CKD. Am J Kidney Dis 2010; 55: 726-741. 
14 Krasuski R, Hart S, Smith B, et al. Association of anaemia and longterm survival in patients with pulmonary hypertension. Int $J$ Cardiol 2010; (In press).

15 World Health Organization. Technical Report Series No. 405. Nutritional Anaemias: Report of a WHO Scientific Group World Health Organization. Geneva, World Health Organization, 1968. http://whqlibdoc.who.int/trs/WHO_TRS_405.pdf.

16 Opasich C, Cazzola M, Scelsi L, et al. Blunted erythropoietin production and defective iron supply for erythropoiesis as major causes of anaemia in patients with chronic heart failure. Eur Heart J 2005; 26: 2232-2237.

17 Nanas JN, Matsouka C, Karageorgopoulos D, et al. Etiology of anaemia in patients with advanced heart failure. J Am Coll Cardiol 2006; 48: 2485-2489.

18 Silverberg DS, Wexler D, Palazzuoli A, et al. The anaemia of heart failure. Acta Haematol 2009; 122: 109-119.

19 Grzeslo A, Jankowska EA, Witkowski T, et al. Iron deficiency is a common finding in patients with stable chronic heart failure. Eur J Heart Fail Suppl 2006; 5: 132.

20 Murphy CL, Fitzsimons EJ, Jardine AJ, et al. Routine assessment of iron status in all patients with heart failure may identify those at risk of developing anaemia. Eur J Heart Fail Suppl 2007; 61: 24.
21 Opitz CF, Kirch W, Mueller EA, et al. Bleeding events in pulmonary arterial hypertension. Eur J Clin Invest 2009; 39: Suppl. 2, 68-73.

22 Humbert M, Monti G, Brenot F, et al. Increased interleukin-1 and interleukin-6 serum concentrations in severe primary pulmonary hypertension. Am J Respir Crit Care Med 1995; 151: 1628-1631.

23 Dorfmuller P, Perros F, Balabanian K, et al. Inflammation in pulmonary arterial hypertension. Eur Respir J 2003; 22: 358-363.

24 Nemeth E, Ganz T. The role of hepcidin in iron metabolism. Acta Haematol 2009; 122: 78-86.

25 Ganz T. Hepcidin, a key regulator of iron metabolism and mediator of anaemia of inflammation. Blood 2003; 102: 783-788.

26 Selimovic N, Bergh CH, Andersson B, et al. Growth factors and interleukin-6 across the lung circulation in pulmonary hypertension. Eur Respir J 2009; 34: 662-668.

27 Smith TG, Balanos GM, Croft QPP, et al. The increase in pulmonary arterial pressure caused by hypoxia depends on iron status. J Physiol 2008; 586: 5999-6005.

28 Smith TG, Talbot NP, Privat C, et al. Effects of iron supplementation and depletion on hypoxic pulmonary hypertension: two randomized controlled trials. JAMA 2009; 302: 1444-1450. 